\title{
Study of PCFs Output Intensity Profiles with Various Configurations for Low $d / A$ Values
}

\author{
A. Zendehnam ${ }^{1}$, M. Hosseinpour ${ }^{1 *}$, M. Mirzaei $^{1}$, and K. Hedayati ${ }^{2}$ \\ ${ }^{1}$ Department of Physics, Faculty of Science, Arak University, Arak, P.O. Box: 38156 - 8-8349, Iran \\ ${ }^{2}$ Departments of Science, Arak University of Technology, University Street, Arak, P.O. Box: \\ 41167-38181, Iran
}

Received 7 November 2013, accepted in a revised form 7 April 2014

\begin{abstract}
In this study, a Gaussian amp function relating to the Gaussian family is employed to approximate the output intensity profile of various arrangements of air holes in photonic crystal fibers (PCFs) with small $d / \Lambda$ values. The studied structures include six distinctive designs starting with square lattice and carried on up to the PCF with Nano configuration. It is shown that $d / \Lambda=0.4$ is the best value of low $d / \Lambda$ ratios $(0.2 \leq d / \Lambda \leq 0.4)$ which leads to the rise of the central intensity noticeably and the reduction of the full width at half maximum at three studied wavelengths $(\lambda=1.35,1.55$ and $1.65 \mu \mathrm{m})$.
\end{abstract}

Keywords: Photonic crystal fibers; Central intensity; Gaussian function.

(C) 2014 JSR Publications. ISSN: 2070-0237 (Print); 2070-0245 (Online). All rights reserved. doi: http://dx.doi.org/10.3329/jsr.v6i2.16901 J. Sci. Res. 6 (2), 193-203 (2014)

\section{Introduction}

Photonic crystal fibers (PCFs) classified according to their core, which is solid or air, are considered to be a micro-structured optical fiber (MSOF) or holey fibers (HF). These fibers not only can be exceled to perform very well in non-telecom or telecom wavelength range, but also they exhibit unique properties [1-7] .This innovative class of fibers are fabricated by embedding a number of air channels which usually form square-lattice or hexagonal-lattice $[8,9]$ around the core and run along the fiber .

These unique characteristics that provide the possibility of varying PCFs ability to concentrate the mode field in the core region by inducing a small change on air-hole structure, have made these fibers to be more interesting than conventional fibers . To illustrate the dependency of PCFs performance and parameters such as the air hole diameter $(d)$ or the lattice constant $(\Lambda)$, recently, comprehensive designs which have proposed several distinctive amounts for these parameters, have been studied [10-12].

\footnotetext{
*Corresponding author: maryamhosseinpour35@yahoo.com
} 
Indeed, in these studies, only specific conditions which may have caused manufacturers some trouble for fabricating PCFs have been opted for effective parameters like $d$ or $\Lambda$.

Moreover, previous studies have focused on the effects of varying the geometrical parameters like the air-filling fraction ratio $(d / \Lambda)$ by rising $d$ or $\Lambda$ on the mode field profile. But it should be noted that in these papers, only one or two special structures such as square and hexagonal designs for telecom [8, 10, 13] and non-telecom [14, 15] wavelengths have been studied. In some previous articles, the mode profiles of PCFs have been fitted approximately by the use of Gaussian and Sech functions [13] as well as with a constructed function [16] but in these reports, it has not emphasized that the results are restricted to PCFs with one or two specific structures ( square and hexagonal designs). Exchanging the circular air holes for square holes $[17,18]$ is another innovative way for improving the ability of PCFs, however, it seems that PCFs with these unique designs can be merely beneficial under special supposed conditions also fabricating these fibers may be uneconomical and very difficult. The significance of the study of output intensity profiles including the central intensity and the width of profiles, appear especially in medical applications such as optical coherence tomography (OCT) imaging [19]. Moreover, it is important to examine the performance of these fibers when the employed wavelength $(\lambda)$ is varied. For example, the dentistry OCT imaging is performed at $\lambda=$ $1.35 \mu \mathrm{m}[20]$. Also, it is reported that OCT imaging at $1.5 \mu \mathrm{m}$ up to $1.6 \mu \mathrm{m}$ broadband light source can be used to take images of human tooth samples [21]. In our early work on PCFs, the confinement loss (CL) and group velocity dispersion (GVD) of two types of MOFs against wavelength were investigated [22]. Moreover, in our previous works [23, 24 , and 25], the effects of various configurations and different wavelengths $(1.2 \mu \mathrm{m} \leq \lambda \leq$ $1.8 \mu \mathrm{m})$ on the intensity distribution, output intensity profiles and confinement loss in PCFs were discussed. Furthermore, in our recent work [26], optimum values of air filling fraction $(d / \Lambda)$ for photonic crystal fibers with different configurations and fixed number of air rings $(N=4)$ were presented. In the present work, we propose a Gaussian amp function for approximating the output intensity profiles of different configuration of PCFs with small air-filling fractions $(d / \Lambda=0.3$ and 0.4$)$ when $\lambda=1.35,1.55$ and $1.65 \mu \mathrm{m}$. The output profiles are fitted well to the proposed function. When $d / \Lambda=0.4$, PCFs with heptagonal, octagonal and Nano configurations offer the maximum output intensity. The highest output intensity (nearly $12 \times 10^{17} \mathrm{w} / \mathrm{m}^{2}$ ) is offered by the nano structure with $d / \Lambda=0.4$ at $\lambda=1.35 \mu \mathrm{m}$ (the lowest operating wavelength).

\section{Results and Discussions}

The numerical results are divided into two sections for $d / \Lambda=0.3$ and $d / \Lambda=0.4$, respectively, to illustrate the effect of each low $d / \Lambda$ values on the intensity profiles. Please note that in the following steps, the number of air rings $(N)$ are kept constant $N=4$ which is found to be a minimum good value for this parameter [23].

When $N=4$, the output intensity is markedly concentrated in the core, whereas, for $N$ $\leq 3$, the tail of the confined intensity extends into the cladding which results in the 
emergence of side dips on the intensity profile. Furthermore, such dips also can be seen in the output profile of the PCFs with the extra number of edges $(n>9)$ in the cladding. Despite raising the output intensity, these PCFs cannot be useful due to the fact that such structures offer the large amounts of $C L$ [23]. Therefore, the PCF with nano configuration is the final studied structure.

We carried out an analytical method by making the use of the $X Y$ Extract Graph Digitizer v5.1 and Origin Pro v8.6 software to evaluate approximately the simulated output intensity profiles. Indeed, in the present work, the COMSOL Multiphysics v3.4 software is applied to simulate the PCFs. All the output profiles obtained in the preceding have been digitized using the $X Y$ Extract Graph Digitizer v5.1 software to find the coordinates of the simulated profiles with great accuracy. These obtained dots are considered as digitized data. In the following step, the digitized data of actual profiles are then approximated using the analytical software, Origin Pro v8.6, with Gaussian amp function which is defined by Eq. (1) [27]:

$$
I(x)=I_{o} \mathrm{e}^{\frac{-x^{2}}{2 w^{2}}},
$$

where $I o$ is the central intensity, the parameter $w$ is standard deviation and $x$ is a position of each point which intensity is calculated for that point (in micron).

Please note that here, we ignore investigating the output intensity profiles of designs with $d / \Lambda=0.2$. According to our results [23], $d / \Lambda=0.2$ leads to the leaky intensity distribution in the cladding region especially for PCFs with more number of edges in the cladding. Moreover, the parameter full width at half maximum $(F W H M)$ which is an important parameter is found by employing the Origin Pro software to estimate the width of output profile. The values obtained for this parameter are more nearly to the amounts which are found with the relationship that has been employed by Wolfram Math World [28].

\subsection{Study of the output intensity profiles belonged to PCFs with various configurations when $d / \Lambda=0.3$}

In the following sections, the output intensity capabilities of the six investigated PCFs with different configurations starting with the square lattice and carried on up to the Nano structure will be discussed when the number of air rings is kept constant $(N=4)$. Fig. 1 shows cross-sections of the examined PCFs. All the fiber designs proposed in the following were simulated according to the building instruction for equilateral polygons based on ref. [29]. Please note that the shape of air-holes for all the examined fibers is circular. Moreover, the arrangement of air holes at the first ring is assumed to be the repeating unit cell. Additionally, in the four ring design, the radius of the second, third and fourth air rings is respectively twice, three and four times larger than the radius of the first ring which has been supposed equal to $2.3 \mu \mathrm{m}$. 

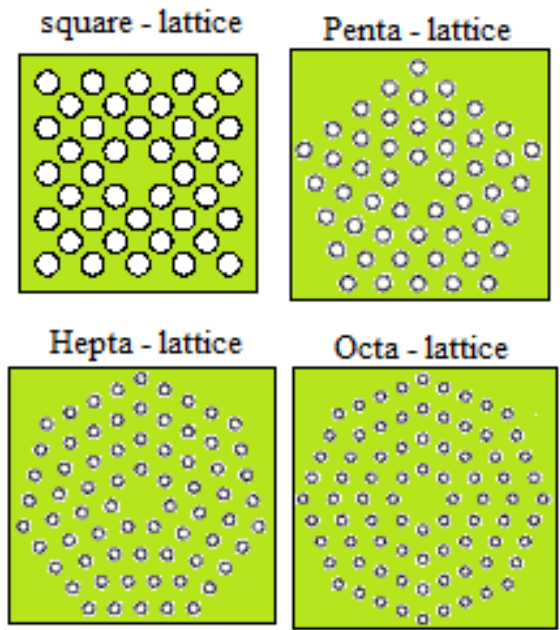

Hexa - lattice

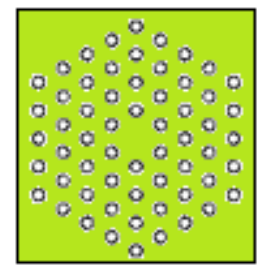

Nano - lattice

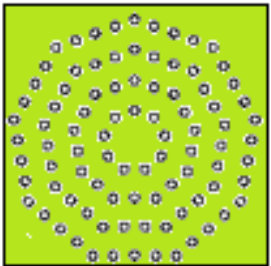

Fig. 1. Cross-sections of the solid core PCFs with various configurations. The white dots represent the air holes in the silica matrix with refractive index $n_{\text {clad }}=1.44$.

The two dimensional (2D) intensity distributions related to all of the simulated PCFs with $d / \Lambda=0.3$ are given in Fig. 2 at $\lambda=1.55 \mu \mathrm{m}$.
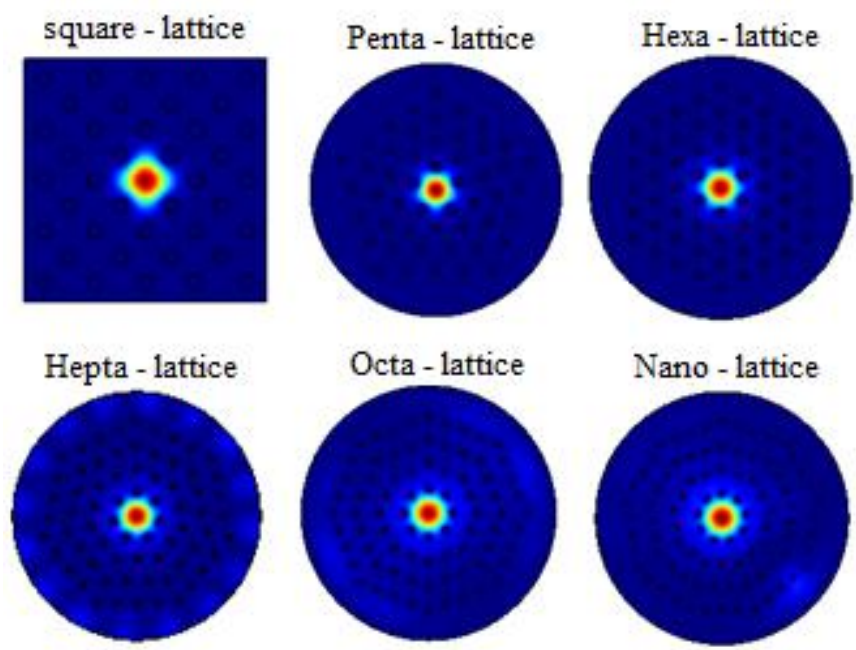

Fig. 2. Simulated 2D intensity distributions of the PCFs investigated at $\lambda=1.55 \mu \mathrm{m}, d / \Lambda=0.3$ and $N=4$. 
As can be seen in Fig. 2, when $d / \Lambda=0.3$, the leaky intensity in the cladding increases as the number of edges rises. The reason for this behavior is that the dimension of air holes $(d)$ becomes far from the employed wavelengths as the number of edges increases.

Fig. 3 shows (a) the two dimensional (2D) intensity distributions (on the left hand side) and (b) intensity profiles of the PCFs considered with symmetrical designs when $d / \Lambda$ $=0.3, N=4$ at $\lambda=1.35 \mu \mathrm{m}$ which all are obtained using the COMSOL software. These profiles are then approximated using the proposed function with high accuracy and illustrated on the right hand side of Fig. 3 [see Fig. 3(c)]. Note that these approximated profiles are exactly the results obtained using the analytical software (Origin Pro). The results obtained for asymmetrical configurations are also shown in Fig. 4.

(a)

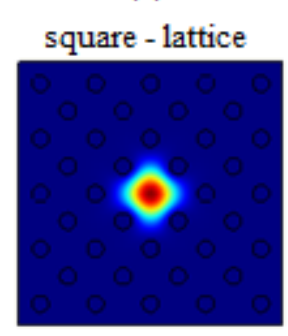

$-10$
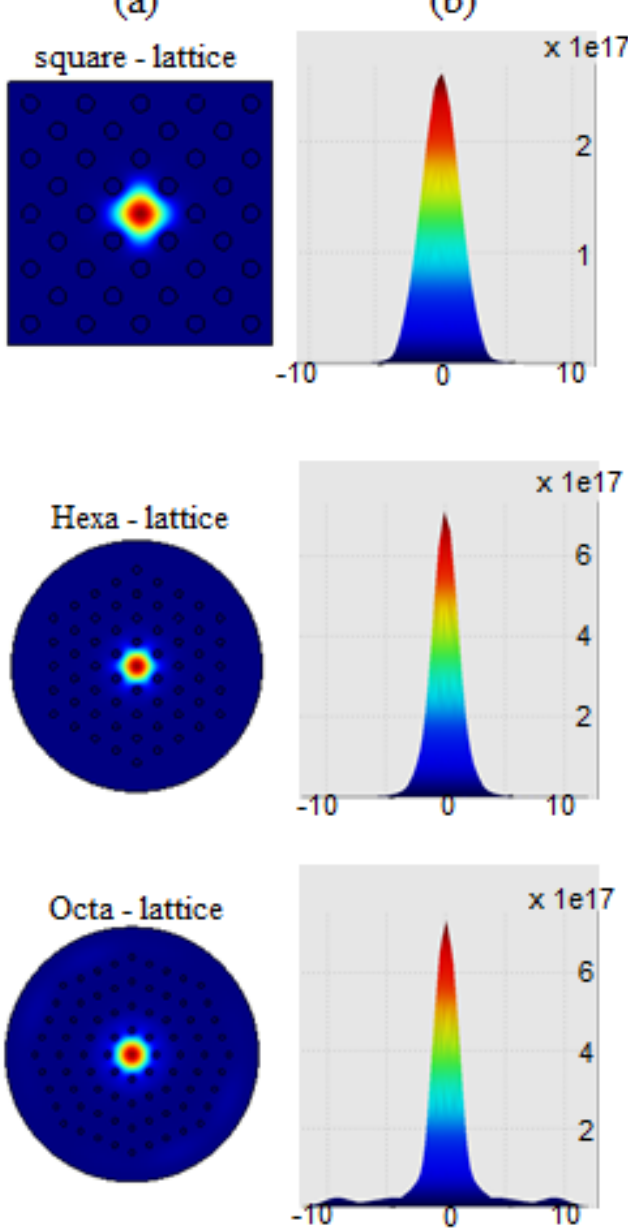

(c)
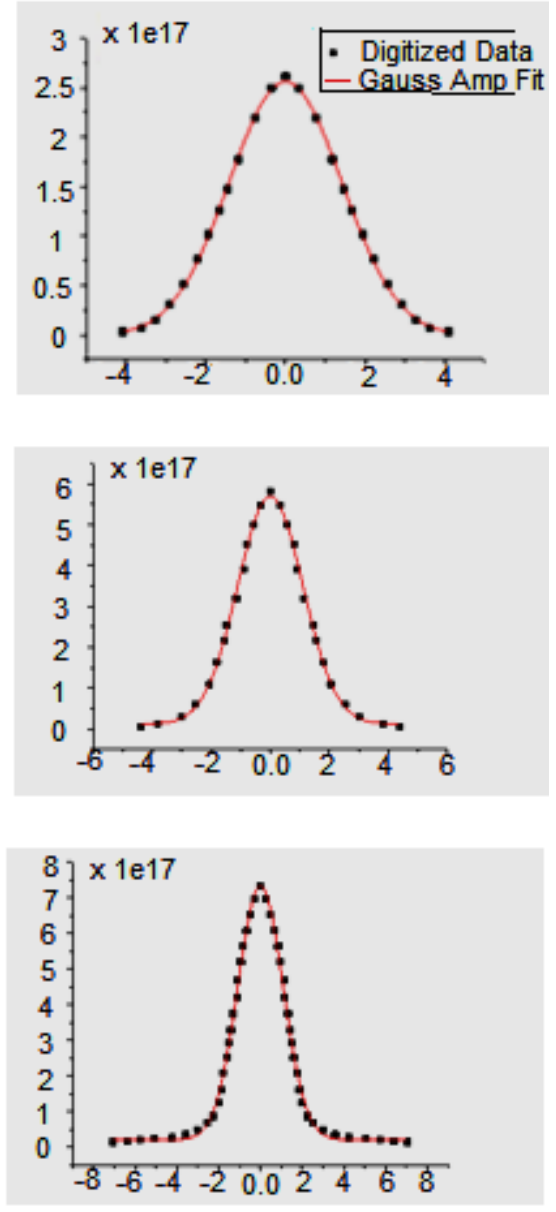

Fig. 3. Simulated 2D (a) intensity distributions and (b) output intensity profiles obtained using COMSOL software, (c) approximated output intensity profiles related to the PCFs with symmetrical designs while $d / \Lambda=0.3, N=4$ and $\lambda=1.35 \mu \mathrm{m}$. 
(a)
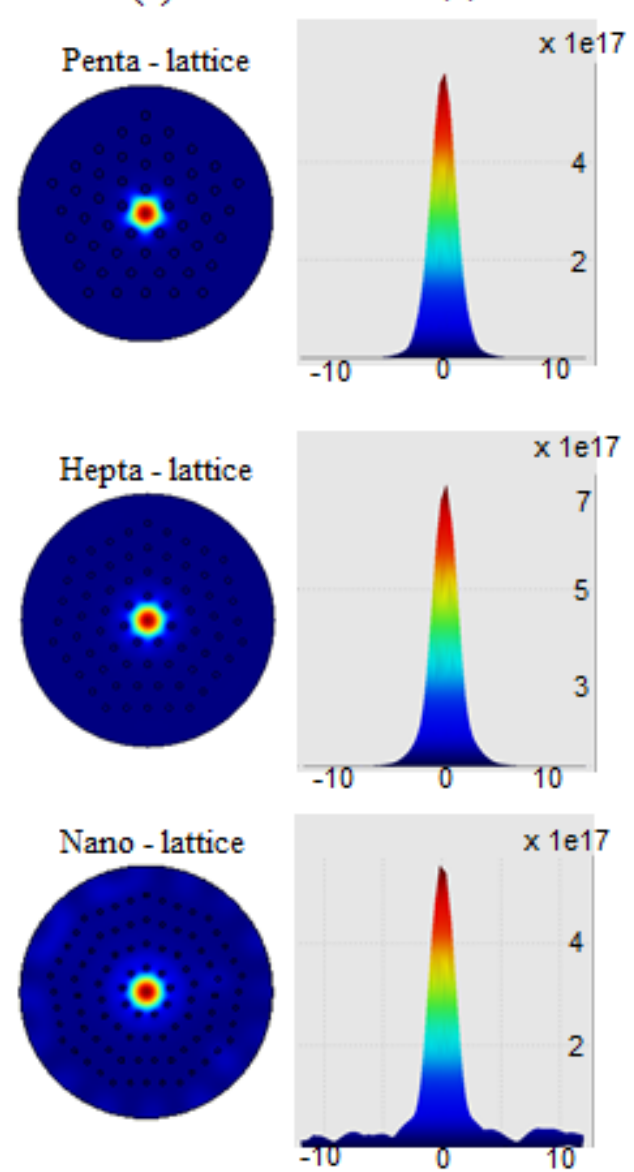

(c)
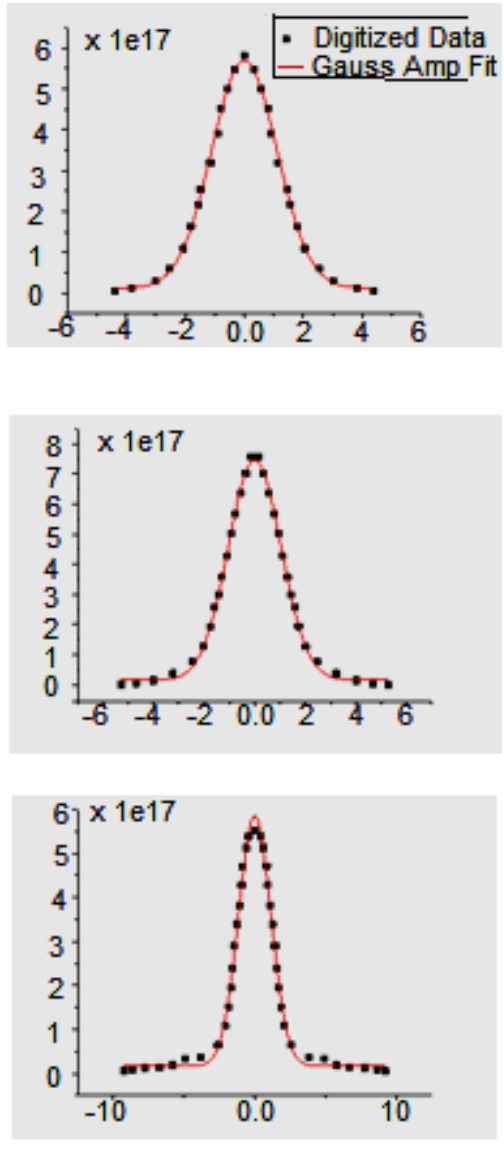

Fig. 4. Simulated 2D (a) intensity distributions and (b) output intensity profiles obtained using COMSOL software, (c) approximated output intensity profiles related to the PCFs with asymmetrical designs while $d / \Lambda=0.3, N=4$ and $\lambda=1.35 \mu \mathrm{m}$.

It is to be noted that in Fig. 3 (b), (c) and Fig. 4 (b), (c) the vertical axis refers to intensity $\left(\mathrm{w} / \mathrm{m}^{2}\right)$ and the horizontal axis represents cross - sections of the examined PCFs (in micron). By looking at Fig. 3 and Fig. 4, it is clear that increasing the number of edges in the cladding from the square lattice to octagonal design raise the central intensity. However, for $d / \Lambda=0.3$, when air - hole geometry is varied from octagonal configuration to nano lattice, the central intensity decreases. The reason for this behavior can be due to the greater leaky intensity in the cladding of the nano structure (see Fig. 4). This leads to the broadening of the output intensity related to the PCF with Nano lattice. Figs. 3 and 4 demonstrate that the digitized data (solid dots) of the simulated PCFs fit fell to the 
Gaussian function (solid curve) when the parameter $n$ (the number of edges) is increased. This approximation is also accurate as the operating wavelength $(\lambda)$ is varied from $1.35 \mu \mathrm{m}$ to $1.65 \mu \mathrm{m}$ (see Fig. 5).

Figs. 5(a) and 5(b) summarize the dependency of the central intensity and the number of edges in the cladding $(n)$ as well as the relationship between the parameter FWHM and $n$ (number of edge) when $d / \Lambda=0.3$ for $\lambda=1.35 \mu \mathrm{m}, 1.55 \mu \mathrm{m}$ and $1.65 \mu \mathrm{m}$. The errors presented by the software which are involved with the approximation shown as error bars in Fig. 5.
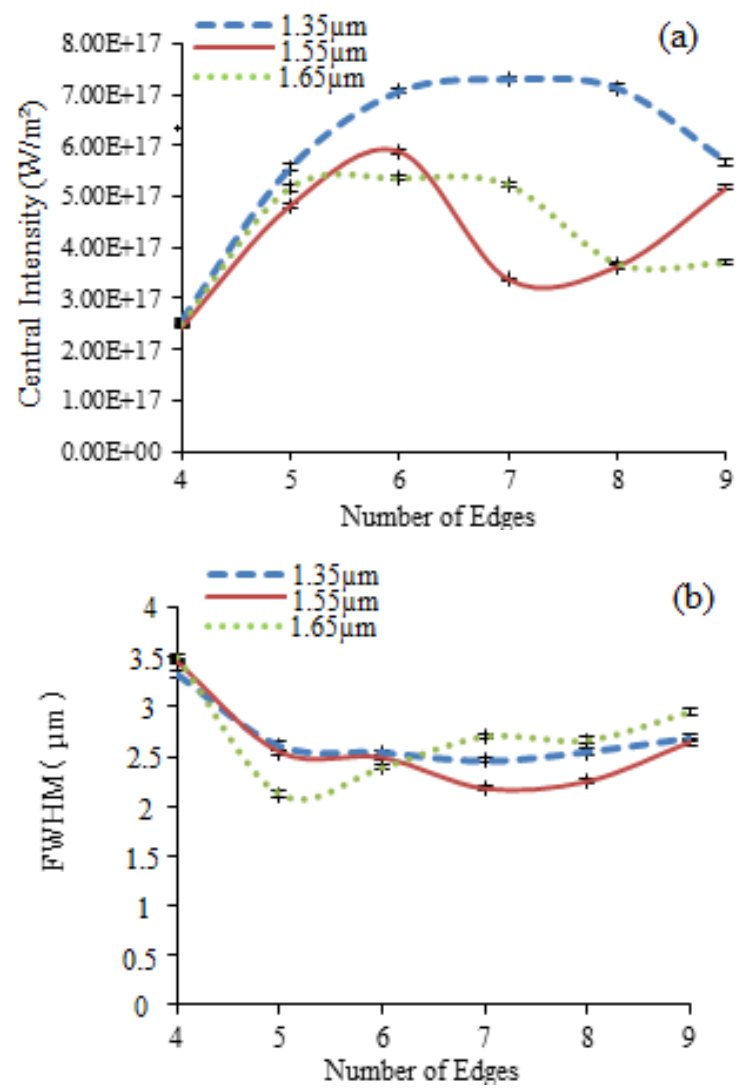

Fig. 5. Plots of a) Central intensity and b) FWHM against the number of edges in cladding region at three wavelengths when $d / \Lambda=0.3$ and $N=4$.

Fig. 5(a) reveals that for $\lambda=1.35 \mu \mathrm{m}$, the PCFs with hexagonal, heptagonal and octagonal designs allow for a central intensity exceeding $7 \times 10^{17} \mathrm{w} / \mathrm{m}^{2}$. In contrast, this increasing behavior occurred in the central intensity plots cannot be seen at the operating wavelengths $\lambda=1.55 \mu \mathrm{m}$ and $1.65 \mu \mathrm{m}$. It is predicted that the dimension of air holes 
especially in the PCFs considered with more number of edges become far from the employed wavelength, when $\lambda$ is increased to $1.55 \mu \mathrm{m}$ and $1.65 \mu \mathrm{m}$. Varying the air - hole geometry from square lattice to nano structure reduces the parameter FWHM at three used wavelengths.

\subsection{Study of the output intensity profiles of PCFs with various configurations while d/A $=0.4$}

The approximated output intensity profiles related to the square and nano designs with $d / \Lambda$ $=0.4$ (the PCFs with the lowest and largest number of edges, respectively) are shown in Fig. 6 and Fig. 7 at three used wavelengths, respectively. According to these figures, it is obvious that the approximation is still accurate when $d / \Lambda=0.4$.
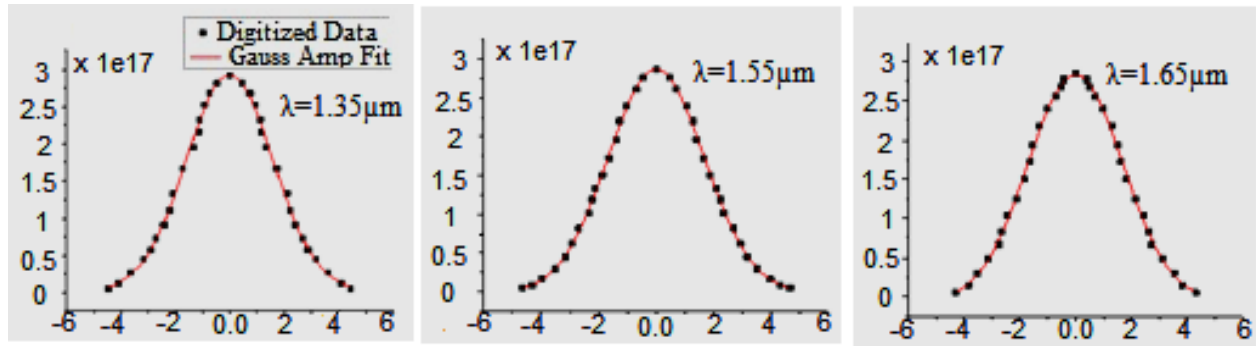

Fig. 6. Approximated output intensity profiles related to the PCF with square lattice for $d / \Lambda=0.4$ when $\lambda$ is varied. The vertical axis refers to intensity $\left(\mathrm{w} / \mathrm{m}^{2}\right)$ and the horizontal axis represents crosssections of the examined PCFs (in micron).
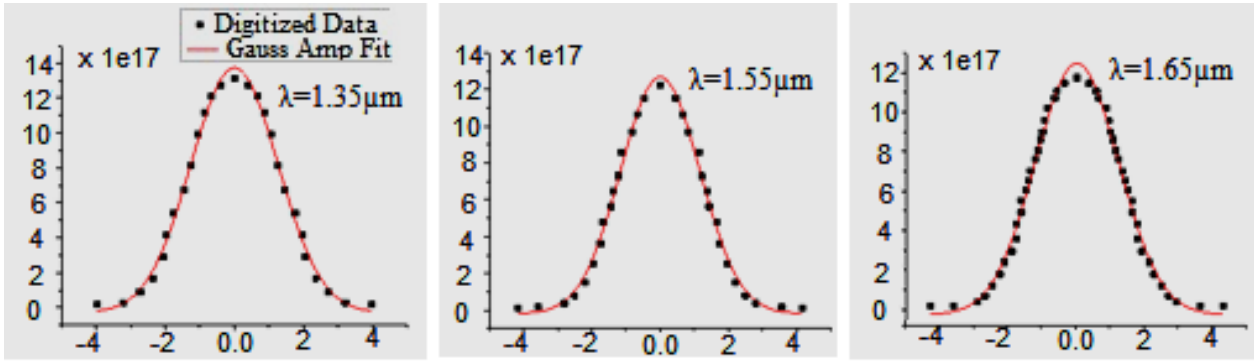

Fig. 7. Approximated output intensity profiles related to the PCF with nano lattice for $d / \Lambda=0.4$ when $\lambda$ is varied. The vertical axis refers to intensity $\left(\mathrm{w} / \mathrm{m}^{2}\right)$ and the horizontal axis represents cross - sections of the examined PCFs (in micron).

Furthermore, plots of the central intensity and parameter FWHM against the number of edges in the cladding are shown in Fig. 8. 

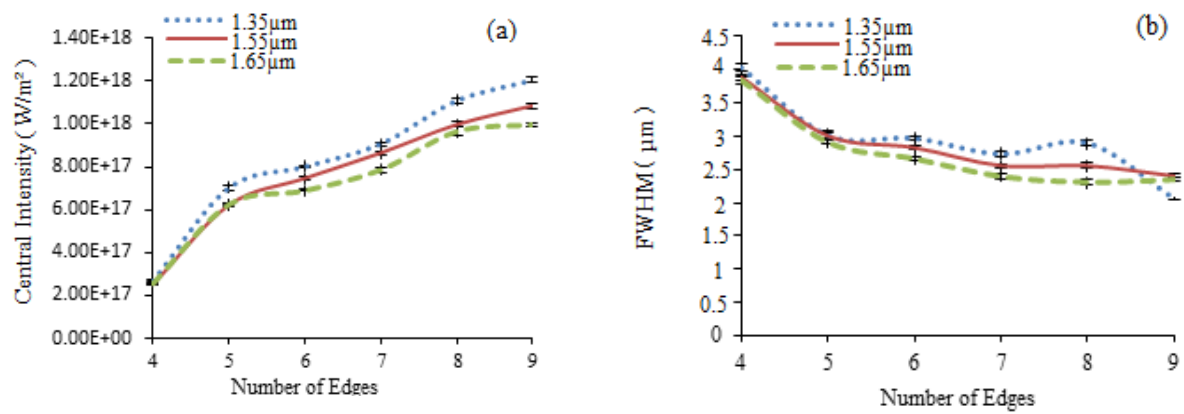

Fig. 8. Plots of a) the central intensity and b) $F W H M$ against the number of edges in cladding region at three wavelengths when $d / \Lambda=0.4$ and $N=4$.

The results of Fig. 7 and Fig. 8 illustrate that for $d / \Lambda=0.4$, the PCF with nano structure, which its number of edges in the cladding is more than other studied configurations, not only boost the central intensity considerably but also decrease the parameter FWHM at $\lambda=1.35 \mu \mathrm{m}, 1.55 \mu \mathrm{m}$, and $1.65 \mu \mathrm{m}$. When $d / \Lambda=0.4$, increasing the number of edges leads to the rise of central intensity continuously. This leads to the strong dependency of the output intensity and the number of air holes in the cladding. Indeed, when $d / \Lambda=0.4$, the relationship between the central intensity and the parameter $n$ shows a sort of nonlinear increase (a sort of polynomial behavior considering the error bars).

It is interesting to note that when $d / \Lambda=0.4$, the marked increment in the central intensity plots as well as the remarkable decrease in the FWHM curves occur exactly where the air-hole geometry is varied from square structure to pentagonal design. Indeed, by considering the data of Table 1 , this behavior can be due to for $d / \Lambda=0.4$, the radius of air holes $(r=d / 2)$ in the PCF with pentagonal $(r=0.5404 \mu \mathrm{m})$ are smaller than the half of employed wavelengths $(\lambda / 2=0.675 \mu \mathrm{m}, 0.775 \mu \mathrm{m}$ or $0.825 \mu \mathrm{m})$. It is to be noted that in the PCF with square design the parameter $r$ is equal to $0.919 \mu \mathrm{m}$ for $d / \Lambda=0.4$.

Table 1. Radius of air holes ( $r$ ) for different configurations when $d / \Lambda$ is fixed on 0.3 and 0.4 , respectively.

\begin{tabular}{ccc}
\hline \multirow{2}{*}{ Structures } & \multicolumn{2}{c}{$r(\mu \mathrm{m})$} \\
\cline { 2 - 3 } & $d / \Lambda=0.3$ & $d / \Lambda=0.4$ \\
\hline Square & 0.689 & 0.919 \\
pentagonal & 0.4053 & 0.5404 \\
Hexagonal & 0.345 & 0.46 \\
Heptagonal & 0.2995 & 0.3995 \\
Octagonal & 0.264 & 0.352 \\
Nano & 0.236 & 0.3145 \\
\hline
\end{tabular}


When $\lambda$ is varied from $1.35 \mu \mathrm{m}$ to $1.65 \mu \mathrm{m}$, the parameter $d$ becomes far from the used wavelength, hence, the central intensity value diminishes in all of the considered configurations. Fig. 8(b) illustrates that the decrease in the parameter FWHM value can still be seen where $\lambda$ is increased from $1.35 \mu \mathrm{m}$ to $1.65 \mu \mathrm{m}$, whereas, for the PCF with nano structure when $\lambda$ is changed from $1.55 \mu \mathrm{m}$ to $1.65 \mu \mathrm{m}$, it is not perceived. It is predicted that this behavior is because of the radius of holes in nano lattice $(r=0.314 \mu \mathrm{m})$ is less markedly than the half of both operating wavelengths $\lambda / 2=0.775 \mu \mathrm{m}$ and $0.825 \mu \mathrm{m}$. In our previous work [23], the confinement loss (CL) of PCFs with small $d / \Lambda$ values has been investigated. Accordingly, it is obtained that $d / \Lambda=0.4$ reduces the values of $C L$ more than other small $d / \Lambda$ values. Hence, $d / \Lambda=0.4$ can not only be suitable in boosting the central intensity but also for reducing the amount of $C L$, especially when $\lambda=1.35 \mu \mathrm{m}$ (the lowest used wavelength).

\section{Conclusion}

In this research, a proposed Gaussian amp function for approximating the output intensity profile of different air hole structure of PCF with small $d / \Lambda$ values is employed. For both $d / \Lambda=0.3$ and 0.4 , the simulated output profiles are fitted well to the proposed function. The delivery of intensity in PCFs depends strongly on the air hole configuration in the cladding. Moreover, the impression of $d / \Lambda=0.3$ and 0.4 on decreasing the parameter FWHM values are roughly similar to each other. When $d / \Lambda \leq 0.3$, the PCFs with less number of edges can be suitable due to a weak intensity confinement that occurs in the PCFs with more number of edge.

When $d / \Lambda=0.4$, the continuous increase seen in the central intensity plots demonstrates the strong dependency of the output intensity and the number of edge (the number of air holes). For $d / \Lambda=0.4$, the marked increment in the central intensity plot [e.g. (2.68 to 7.02 ) $\times 10^{17} \mathrm{w} / \mathrm{m}^{2}$, at $\left.\lambda=1.35 \mu \mathrm{m}\right]$ is perceived where the PCF air hole structure is varied from square lattice to pentagonal configuration.

\section{References}

1. D. Ferrarini, L. Vincetti, and M. Zoboli, Opt. EXP. 10, 1314 (2002). http://dx.doi.org/10.1364/OE.10.001314

2. K. Saitoh and M. Koshiba, Opt. Exp.12, 2027 (2004).

3. http://dx.doi.org/10.1364/OPEX.12.002027

3. T. Matsui, J. Zhou, K. Nakajima, and I. Sankawa, J. Lightwave Tech. 23, 4178 (2005).

4. X. Tan, Y. Geng, Z. Tian, P. Wang, and J. Yao, Opt. Lett. 5, 124 (2009). http://dx.doi.org/10.1007/s11801-009-8194-8

5. M. Chen and Sh. Xie, Opt. Commun. 281, 2073 (2008). http://dx.doi.org/10.1016/j.optcom.2007.12.006

6. Nh. Hai, Y. Namihira, F. Begum, S. Kaijage, S. M. A. Razzak, T. Kinjo, and N. Zou, IEICE Trans. Elect. 90, 1627 (2007).

7. S. Lee, W. Ha, J. Park, S. Kim, and K. Oh, Optics Commun. 285, 4082 (2012). http://dx.doi.org/10.1016/j.optcom.2012.05.063

8. H. Demir and S. Ozsoy, Optical Fiber Tech. 17, 594 (2011). http://dx.doi.org/10.1016/j.yofte.2011.08.003 
9. H. Demir and S. Ozsoy, Optik: Intern. J. Light Electr. Optics 28, 257 (2012).

10. S. Olyaee and F. Taghipour, Intern. J. Phys. Sci. 6, 4405 (2011).

11. S. M. Nejad and N. Ehteshami, A novel design to compensate dispersion for square-lattice photonic crystal fiber over E to L wavelength bands, In: Proc. IEEE Conference on Communication Systems Networks and Digital Signal Processing, Newcastle upon Tyne (2010) pp. $654-658$.

12. M. N. Hoque, A. Sayeem, and N. Akter, Aust. J. Basic Appl. Sci. 4, 2274 (2010).

13. T. Hirooka, Y. Hori, and M. Nakazawa, IEEE Photo. Tech. Lett. 16, 1071 (2004). http://dx.doi.org/10.1109/LPT.2004.824950

14. K. N. Park and K. S. Lee, Opt.EXP. 16, 13175 (2008). http://dx.doi.org/10.1364/OE.16.013175

15. M.. O. Faruk, Ma. ul Aftab, R. Ahmed, M. B. Hossain, M. S. Islam, and S. B. Alam, Effect of lattice constant and air hole diameter on the mode profile in triangular and square lattice photonic crystal fiber at $\mathrm{THz}$ regime - Proc. the World Congress on Engineering and Computer Science, pp. 1109-1114 (2010).

16. L. Zhang, Z. Wu, S. Gao, and M. Cui, Optic. Eng. 51, 065003-1 - 065003-11 (2012).

17. H. Demir and S. Ozsoy, Optic. Mater. 35, 205 (2012). http://dx.doi.org/10.1016/j.optmat.2012.07.028

18. H. Demir and S. Ozsoy, Optic. Quant. Electron. 42, 851 (2011). http://dx.doi.org/10.1007/s11082-011-9493-4

19. F. Begum and Y. Namihira, Photonic crystal fiber for medical applications, In: The Recent Progress in Optical Fiber Research In Tech., Dr. Moh. Yasin ed, (2012) pp. 230-246.

20. S. A. Bopart, B. E. Bonma, C. Pitris, J. F. Southern, M. E. Brezinski, and J. G. Fujimoto, Nature Medicine 4, 861 (1998). http://dx.doi.org/10.1038/nm0798-861

21. J. H. Lee, E. J. Jung, and C. Kim, Proc. IEEE Conference on OptoEelectronics and Communication, FD3 (2009).

22. A. Zendehnam, M. Mirzaei, and R. Solgi, Int. J. Optics Appl. 2, 34 (2012).

23. M. Hosseinpour, M.S. Thesis, Arak University, Arak, Iran (code: 2135257) (2013).

24. M. Hosseinpour and A. Zendehnam, The Study of the Influence of PCFs with Various Configurations on Output Intensity Profiles, In: Proc. the 20th Iranian Conf. on Optics and Photonics (ICOP) and the 6th Iranian Conf. on Photonics Eng. Tech. (ICPET) (Shiraz, Iran, 28 30 January 2014).

25. M. Hosseinpour and A. Zendehnam, The Influence of Variation in Effective Parameters on Confinement Loss in Photonic Crystal Fibers, In: Proc. of the 20th Iranian Conference on Optics and Photonics, (ICOP) and the 6th Iranian Conf. on Photonics Eng. Tech. (ICPET) (Shiraz, Iran, 28 - 30 January 2014).

26. A. Zendehnam, M. Hosseinpour, M. Mirzaei, and K. Hedayati, Appl. Optics 53, 1075 (2014). http://dx.doi.org/10.1364/AO.53.001075

27. J. T. Verdeyen, Laser electronics 2, 69 (Science, 1995).

28. W. MathWorld, "Gaussian Function", http://mathworld.wolfram.com/gaussianfunction.html

29. F. Stutzki, F. Jansen, C. Jauregui, J. Limpert, and A. Tünnermann, Opt. EXP. 19, 12081 (2011). http://dx.doi.org/10.1364/OE.19.012081 